\title{
Model of Optical Sensor on the Base of MMI Structures
}

\author{
A. SzewczuK*, M. BŁahut and W. Pyka \\ Department of Optoelectronics, Silesian University of Technology, Krzywoustego 2, 44-100 Gliwice, Poland \\ The paper presents the research on waveguide sensors based on multimode interference structures. In the \\ modeled systems, the applied sensor layer was used as the cover of the multimode section. The cover of the \\ multimode section can be made of material sensitive to the changes of definite external conditions, and through a \\ proper selection of this material the sensor can be adapted to the detection of various physicochemical quantities. \\ In effect of the changes of optical parameters of the sensor layer, the propagation conditions change, which can \\ be easily observed in the location changes of $N$-fold images of the input field. In the paper we have investigated \\ and compared the systems based on waveguides having step index profile and gradient distribution of refractive \\ index. We have demonstrated that the sensitivity of such systems can be adjusted through a proper selection of \\ the dimensions of the multimode section.
}

PACS numbers: 42.79.Gn, 42.82.Ds, 42.79.-e

\section{Introduction}

A growing popularity of waveguide sensors entails the possibility to obtain high sensitivities coupled with the simplicity of operation. The functioning of such devices is usually based on the principle implying that the change of external physical parameters leads to the change of propagation conditions of light, and its effect is easily observable in the output signal. The present paper is devoted to one of the concepts of waveguide structures based on multimode interference (MMI) structures. The results presented in the publications $[1,2]$ show that the modeled devices are characterized by high sensitivity which can be easily shaped through a proper selection of the dimensions of the multimode waveguide. The first paper [1] describes a modeled humidity sensor which can be produced using the planar sol-gel technology. The publication demonstrates the produced prototype device for which we were able to obtain linear changes of the signal in response to the linear changes of relative humidity. It has been demonstrated that the sensitivity of the device produced in line with this concept can be selected through a proper modeling of MMI structures. The second paper presented here [2] offers the results of the simulation for the system modeled as gas sensor. In the publication we have presented the discussion aiming to optimize the sensitivity of such a device, and we have demonstrated that the structures designed in such a way are characterized by much higher sensitivity than the devices based on the Mach-Zehnder interferometer.

The paper presents the operation and performance characteristics of the modeled sensors. It has been

\footnotetext{
* corresponding author; e-mail: artur.szewczuk@polsl.pl
}

demonstrated that the position of input field image depends closely on the optical parameters of the layer covering the multimode waveguide. Two configurations of the sensor have been presented and compared. One of the configurations was based on gradient waveguides produced in ion exchange technology, and the other one was based on waveguides having step index distribution of the refractive index.

\section{Operation principle}

Multimode interference structures are optical elements which consist of three sets of waveguides: single mode input and output waveguides and a multimode waveguide placed between them. The single mode field generated by the input waveguide is decoupled into many modes in the multimode section. Inside the multimode section, the particular modes are propagating at various phase velocity and therefore they are shifted in the phase in a different way [3]. The field distribution at the distance $z$ away from the structure excitation place is described by the following expression:

$$
\begin{aligned}
& E(x, y, z)=\exp \left(-\mathrm{j} \beta_{0} z\right) \\
& \quad \times \sum_{l} c_{l} \varphi_{l}(x, y) \exp \left(-\mathrm{j}\left(\beta_{0}-\beta_{l}\right) z\right) .
\end{aligned}
$$

The phase changes in the above formula refer to the phase of zero mode. For the investigated structures (gradient distribution and step index of the refractive index) the dependence of propagation constants on the mode number can be with high accuracy described by the square function $[4,5]$ :

$$
\beta_{0}-\beta_{l}=\frac{l(l+2) \pi}{3 L_{z}} .
$$

By inserting this dependence to the formula defining the 
value of field intensity at the distance $z$, we obtain the position of the point at which the input field is reproduced. When the value of the exponent in the equation is equal to one, then the field amplitude reflects the amplitude of the input field

$$
\exp \left(\mathrm{j} \frac{l(l+2) \pi}{3 L_{z}} z\right)=1 \Rightarrow L=n\left(3 L_{z}\right) .
$$

Then, the place at which the input field is reproduced is dependent on the difference of propagation constants of the modes $\beta_{0}-\beta_{l}[3]$. The mentioned difference can be influenced through the change of geometrical dimensions, or by changing the values of the refractive indexes of the particular layers of the waveguide. Hence we suggest the concept of covering the multimode core with a sensor layer whose optical parameters depend on external environment (Fig. 1). The shift of the input field image with respect to its primal position will result in the change of the signal propagating in the output waveguide.

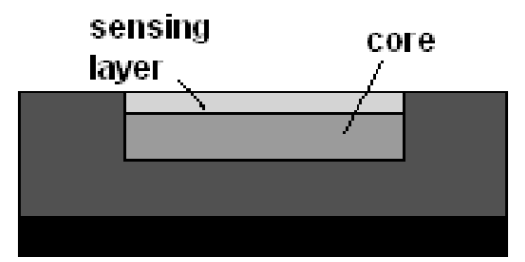

Fig. 1. Diagram of the modeled sensor.

\section{Optical structures}

The analysis of the particular models was based on the numerical method BPM used to analyze the propagation of light in waveguides. The MMI section consists of several layers. The base was made up by a buffer layer whose aim was to isolate the core from the substrate. On the buffer layer, a core lined with the cover layer was placed. A sensor layer deposited directly on the core was built in into the cover. In the case of input and output waveguides, the cover and substrate are made from the same materials as for the multimode structure. The investigation involved the structures produced both in the step index technology and with the gradient distribution of the refractive index.

\subsection{Step index}

The dimensions were selected in the way ensuring that a single mode status of the waveguides (the input and output ones) could be obtained for the wavelength of $6328 \mathrm{~nm}$. The core was $0.8 \mu \mathrm{m}$ thick. The waveguides the input and output ones - were $4 \mu \mathrm{m}$ wide. The refractive index of the substrate was 1.51, and that of the core was 1.52. The refractive index of the sensor layer was being changed within the range of 1.47-1.49. The geometry of the structure was selected in the way ensuring that it could be comparable to gradient structures analyzed in the next point.

\subsection{Gradient index}

For the needs of the carried out investigation studies it was assumed that the designed systems based on gradient waveguides had been produced using the ion exchange technique. This method consists in diffusing of admixture ions to the near-the-surface area of the substrate $[6,7]$. In effect, we bring about the change of physical properties of the near-the-surface area, and, in particular, the change of refractive index. In this process, the substituted ions of the concentration $c_{a}$ replace the ions $c_{b}$ which are natural components of glass substrate. The diffusion equation for the normalized concentration $N_{a}=c_{a} / c_{r}$, where $c_{r}=$ $c_{a}+c_{b}$ has the following form [4]:

$$
\frac{\partial N_{a}}{\partial t}=\nabla\left[\frac{D_{a}}{1-(1-r) N_{a}} \nabla N_{a}\right],
$$

where $r=\mu_{a} / \mu_{b}$ is the ratio of the mobility of ions $(a)$ to the mobility of ions $(b), D_{a}$ is the diffusion index of ions $(a)$.

Basing on the diffusion equation, we numerically calculated the distribution profiles of the refractive index of the waveguides. The ion exchange process $\mathrm{K}^{+}-\mathrm{Na}^{+}$was modeled, for which the following technological parameters of ion exchange were accepted (Table I) [4]. The investigation involved structures of the crosswise dimensions of the multimode section affected by the diffusion through the windows of the width of $40,50,60 \mu \mathrm{m}$, and in the case of the single mode waveguide, of the width $4 \mu \mathrm{m}$.

TABLE

Parameters of diffusion process.

\begin{tabular}{l|c}
\hline \hline$D_{a}\left[\mu \mathrm{m}^{2} / \mathrm{h}\right]$ & 2.18 \\
$r$ & 0.9 \\
diffusion time $t[\mathrm{~h}]$ & 1 \\
$\Delta n$ & 0.0095
\end{tabular}

\section{MMI structures}

\subsection{Symmetrical MMI structure}

The first investigated configuration involved the symmetrical MMI structure of the multimode section width of $50 \mu \mathrm{m}$. We decided to select the length of MMI section to ensure the acquisition of a 1-fold image of the input field. The working point was defined for the refractive index of the sensor layer equal to 1.47. It was essential to establish the influence of external dielectric cover on the images of modal field interference. Figure 2 presents the differences of mode propagation constants calculated with the method of effective refractive index as dependent on the optical parameters of the sensor layer.

As can be observed on the presented characteristic, the difference in mode propagation constants, which determines the image of modal interference, depends on the refractive index of the cover. In consequence, the change of the optical parameters of the sensor layer will bring 


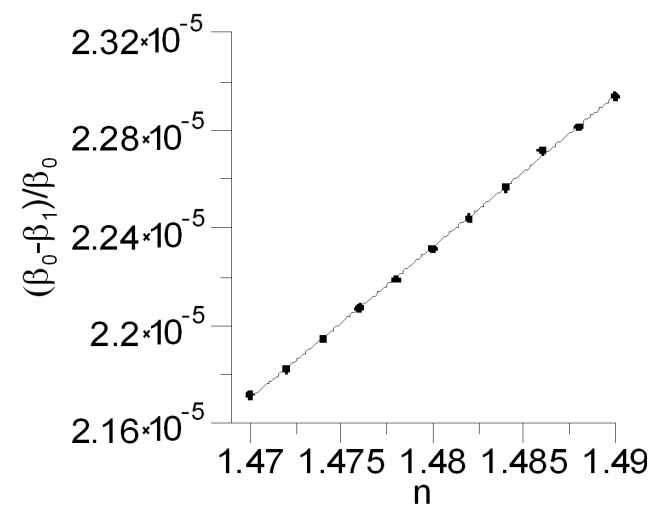

Fig. 2. Dependence of the relative difference of propagation constants between the base mode and the first order mode.
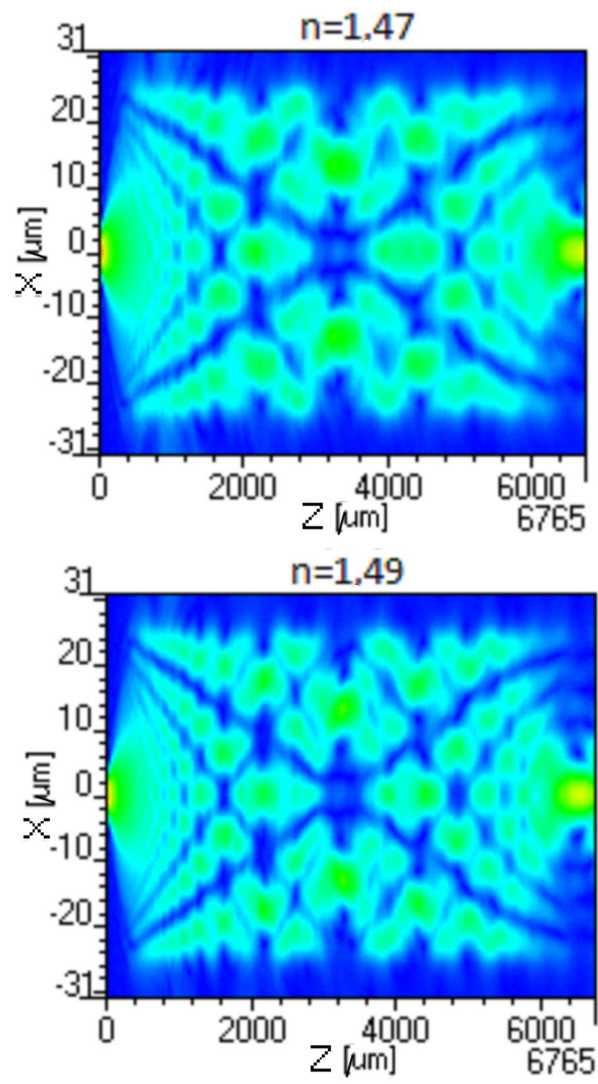

Fig. 3. Field distributions along the propagation path in the multimode core in the structure with refractive index of sensing layer equal to 1.47 (a) and 1.49 (b).

about the shift of the image reproducing the input field (Fig. 3).

Figure 4 presents the dependence of field amplitude at the structure output, obtained on the basis of numerical simulations, as the function of the refractive index of the sensor layer. The simulations were carried out for two different lengths of the multimode section. The lengths were selected in the way ensuring the acquisition of the input field images at the output. In the first case, the length was $6765 \mu \mathrm{m}$ and it ensured the acquisition of the first image of the input field at the output of the multimode section. In the second case, the length was five times higher which ensure the 5 -fold image observation.

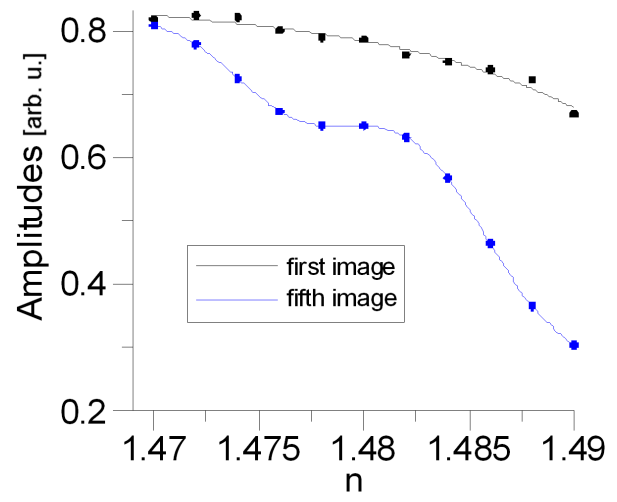

Fig. 4. Dependence of the output amplitude as the function of refractive index of the sensor layer.

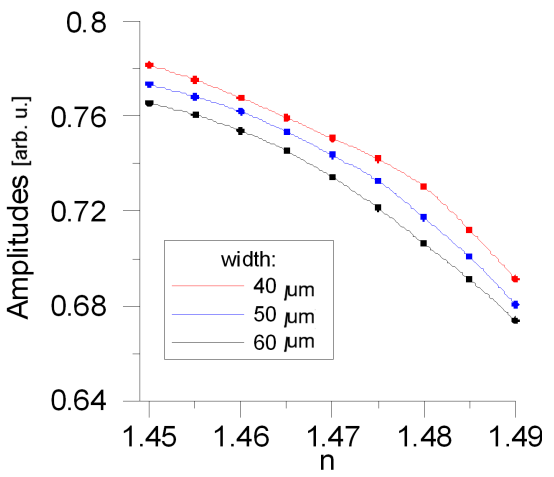

Fig. 5. Dependence of the output amplitude as a function of the refractive index of the sensor layer.

In the case of a longer structure, the changes of the refractive index are accompanied by higher changes of the amplitude than it was observed for a shorter structure (Fig. 5). When the input field is reproduced along the propagation path $d$, then on the path $n d$ it will be reproduced for the $n$-th time. Due to the change of refractive index, the field image is shifting, and the shift of the $n$-th field imaging will be $n$ times higher. Hence, as expected, the larger the order of the reproduced field the higher the sensitivity of the device. The characteristic is not precisely monotonic, but an inflection can be observed. It is connected with the rise of modes number. For the refractive index of 1.48, eight modes can be excited in the structure along the direction consistent with the structure width. For the previous point, allowed for on the characteristic, one mode less is excited. 


\subsection{Rectangular MMI structure of the gradient distribution of refractive index}

In the following stage, the investigation involved the configurations of the sensor with a symmetrical MMI section based on the waveguides produced in the diffusion process. The research was focused on the structures of the multimode section widths of 40,50 and $60 \mu \mathrm{m}$. The refractive index of the sensor layer was being changed within the range of $1.45-1.49$. In effect of the carried out numerical simulations, we obtained the characteristics presenting the dependence of the output signal amplitude as a function of the refractive index of the sensor layer. The length of the multimode sections was selected in the way ensuring the acquisition of input field image at the output for the refractive index of the sensor layer of 1.45 .

The system is characterized by a similar sensitivity as for the system based on planar structures of the step index distribution of the refractive index. The sensitivity does not depend solely on the width of the structure. The relative changes of the amplitude are characterized by the same dynamics for different widths of the multimode waveguide. In the case of wider structures, the number of modes excited in the multimode section is higher and the propagation path is longer after passing which the input field image is obtained. Such structures are also characterized by signal transmission. As presented above, the sensitivity will be dependent on the order of the input field image. It is advisable at the design stage to select the lowest possible width of the structure for which the waveguide will be still a multimode one, affecting at the same time the sensitivity by increasing the length.

\section{Conclusions}

The paper presents different configurations of the waveguide sensor on the basis of multimode interference structures. The basic parameter which is helpful to classify a measuring device is the sensitivity to the changes of the parameters being measured. Having analyzed the results, we can clearly observe that the sensitivity of the devices designed in such a way can be increased through the elongation of the MMI section. Generally speaking, the more reproductions of the input field are involved the higher sensitivity can be achieved.

Comparing the results obtained for step index waveguides with the results obtained for gradient structures we can see that the dynamics of the changes is on the same level. Slightly higher changes of the amplitude are obtained for the step index structures. In the case of gradient structures, a more regular shape of the characteristic is obtained.

\section{Acknowledgments}

The work was sponsored by the State Committee for Scientific Research (NCBiR) within the grant N R01 034 06/2009.

\section{References}

[1] K.R. Kribich, R. Copperwhite, H. Barry, B. Kolodziejczyk, J.M. Sabattie, K. O'Dwyer, B.D. MacCraith, Sensors Actuators $B$ 188, 678 (2005).

[2] T. Mazingue, R.K. Kribich, P. Etienne, Y. Moreau, Opt. Commun. 278, 312 (2007).

[3] L.B. Soldano, E.C.M. Pennings, Lightwave Technol. 13, 615 (1995).

[4] M. Błahut, D. Kasprzak, Opt. Appl. 34, 574 (2004).

[5] T. Pustelny, M. Grabka, Acta Phys. Pol. A 116, 385 (2009).

[6] M. Blahut, D. Kasprzak, M. Sujewicz, Acta Phys. Pol. A 116, 261 (2009).

[7] K. Gut, J. Phys. 129, 109 (2005). 